\title{
Hyperfine structure of S-states of muonic tritium
}

\author{
F.A. Martynenko ${ }^{1, *}$, R.N. Faustov ${ }^{2}$, and A.P. Martynenko ${ }^{1}$ \\ ${ }^{1}$ Samara University, 443086 Samara, Russia \\ ${ }^{2}$ Institute of Informatics in Education, FRC CSC RAS, 119333 Moscow, Russia
}

\begin{abstract}
On the basis of quasipotential method in quantum electrodynamics we carry out a precise calculation of hyperfine splitting of $\mathrm{S}$-states in muonic tritium. The one-loop and two-loop vacuum polarization corrections, relativistic effects, nuclear structure corrections in first and second orders of perturbation theory are taken into account. The contributions to hyperfine structure are obtained in integral form and calculated analytically and numerically. Obtained results for hyperfine splitting can be used for a comparison with future experimental data of CREMA collaboration.
\end{abstract}

A precise calculation of hyperfine splitting of S-states in muonic tritium atom is carried out. Muonic tritium consists of a triton and a negatively charged muon. Its lifetime is determined by the lifetime of the muon $\tau=2.19703940 \cdot \mu \mathrm{s}$. Measuring the frequency of transition (2P-2S) in muonic hydrogen, helium ions with an accuracy of $50 \mathrm{ppm}$ will yield the value of the charge radius of the proton, deuteron, triton, helion and $\alpha$-particle with an accuracy of $0.0005 \mathrm{fm}[1,2]$. For comparison between theory and experiment it is necessary to perform analytical and numerical calculation of the corrections of the fifth and sixth order over the fine structure constant in the hyperfine structure (HFS) of S-states of muonic tritium within the quasipotential method in quantum electrodynamics. Muon atoms differ from electron atoms that for them there is a wide class of corrections to the energy spectrum, which should be examined directly for each muon atom.

On the basis of quasipotential method [3-5] the two-particle bound state is described by the Schrödinger equation. The main contribution to the interaction operator of a muon and triton is determined by the Breit Hamiltonian:

$$
\begin{gathered}
H_{B}=H_{0}+\Delta V_{B}^{f s}+\Delta V_{B}^{h f s}, H_{0}=\frac{p^{2}}{2 \mu}-\frac{Z \alpha}{r}, \\
\Delta V_{B}^{f s}=-\frac{p^{4}}{8 m_{1}^{3}}-\frac{p^{4}}{8 m_{2}^{3}}+\frac{\pi Z \alpha}{2}\left(\frac{1}{m_{1}^{2}}+\frac{1}{m_{2}^{2}}\right) \delta(\mathbf{r})-\frac{Z \alpha}{2 m_{1} m_{2} r}\left(p^{2}+\frac{\mathbf{r}(\mathbf{r p ~}) \mathbf{p}}{r^{2}}\right), \\
\Delta V_{B}^{h f s}=\frac{8 \pi \alpha \mu_{t}}{3 m_{1} m_{p}} \frac{\mathbf{\sigma}_{1} \boldsymbol{\sigma}_{2}}{4} \delta(\mathbf{r}),
\end{gathered}
$$

where $m_{1}, m_{2}$ - masses of muon and triton, $m_{p}$ - proton mass, $\mu_{t}$ - triton magnetic moment.

The potential (3) gives the leading order contribution to hyperfine structure (the Fermi energy). One part of corrections to hyperfine structure is known in analytical form from calculations in electronic hydrogen [6]. They can be used to estimate the contributions to the HFS of muonic tritium. But there are effects that are specific to each of the muon atom.

\footnotetext{
*Corresponding author: f.a.martynenko@gmail.com
} 
One such effect is the effect of vacuum polarization, which leads to an additional term in the Hamiltonian

$$
\Delta V_{1 \gamma, V P}^{h f s}(r)=\frac{8 \alpha \mu_{t}}{3 m_{1} m_{p}} \frac{\boldsymbol{\sigma}_{1} \boldsymbol{\sigma}_{2}}{4} \frac{\alpha}{3 \pi} \int_{1}^{\infty} \rho(s) d s\left[\pi \delta(r)-\frac{m_{e}^{2} \xi^{2}}{r} e^{-2 m_{e} s r}\right],
$$

The potential (4) gives the contribution to HFS of order $\alpha^{5}$ after averaging over the wave functions. The same order contribution is determined by muon anomalous magnetic moment. The two-loop vacuum polarization potentials are also considered. In this work we take into account one-loop and two-loop corrections on the vacuum polarization in first and second orders of perturbation theory. Relativistic corrections of six order in $\alpha$ are included in total value of hyperfine splitting.

Nuclear structure and recoil effects are determined in the leading order by two-photon exchange amplitudes. For their calculation the triton vertex operator is parameterized by two electromagnetic form factors, and the contribution of these amplitudes to the hyperfine structure is represented in integral form and calculated numerically. To construct the interaction operator from two-photon exchange amplitudes with the account of radiative corrections we use a method of projection operators on two-particle states with definite spin $\mathrm{S}=0,1$. The introduction of such projection operators allows further to use package Form [7] to calculate the trace of the product of the Dirac matrices and convolutions by Lorentz indices [8-9]. Numerical contributions of $2 \gamma$-amplitudes to HFS are obtained by means of the Gaussian and dipole parameterizations for the triton form factors. The most significant theoretical error is related namely with $2 \gamma$-amplitudes. We calculate also more complex corrections which include both nuclear structure and vacuum polarization effects.

To calculate different corrections in second order perturbation theory we use a compact representation for the reduced Coulomb Green's function obtained in [10]. To calculate numerous matrix elements of interaction operator (coordinate integration) we use a system Mathematica. The values of fundamental physical constants (particle masses, fine structure constant, muon anomalous magnetic moment, triton charge radius) are taken from [11-12]. Total more precise values of hyperfine splittings are obtained: $(237.667 \pm 0.085) \mathrm{meV}(1 \mathrm{~S}-$ state) and (29.703 \pm 0.011$) \mathrm{meV}$ (2S-state), which can be used for a comparison with future experimental data of CREMA (Charge Radius Experiments with Muonic Atoms) collaboration [1-2]. Performed precise calculation of the hyperfine structure of the muonic tritium is also important to calculate the formation rate of mesic molecules $(p t \mu),(d t \mu)$ and flow of muon catalyzed reactions [13].

The support from Russian Foundation for Basic Research (grant 16-02-00554) and Ministry of Education and Science of Russia (grant 1394) is acknowledged.

\section{References}

1. R. Pohl, A. Antognini, F. Nez et al., Nature 466, 213 (2010)

2. J.J. Krauth, M. Diepold, B. Franke et al., Ann. Phys. 366, 168 (2016)

3. A.P. Martynenko, J. Exp. Theor. Phys. 106, 690 (2008)

4. R.N. Faustov, A.P. Martynenko, J. Exp. Theor. Phys. 88, 672 (1999)

5. R.N. Faustov, A.P. Martynenko, J. Exp. Theor. Phys. 98, 39 (2004)

6. M.I. Eides, H. Grotch, V.A. Shelyuto, Phys. Rep. 342, 62 (2001)

7. J.A.M. Vermaseren, e-preprint arXiv:math-ph/0010025

8. R.N. Faustov et al., Phys. Lett. B 733, 354 (2014)

9. R.N. Faustov et al., Phys. Rev. A 90, 012520 (2014)

10. H.F. Hameka, J. Chem. Phys. 47, 2728 (1967)

11. P.J. Mohr, B.N. Taylor, D.B. Newell, Rev. Mod. Phys. 84, 1527 (2012)

12. Angeli, K.P. Marinova, Atom. Data Nucl. Data Tables 99, 69 (2013)

13. S.S. Gershtein, Yu.V. Petrov, L.I. Ponomarev, Sov. Phys. Usp. 33, 591 (1990) 